\title{
LA INFLUENCIA DEL LATÍN EN LA PUNTUACIÓN DE NEBRIJA
}

\author{
Aneta Pawlak ${ }^{1}$ \\ https://doi.org/10.18778/8220-421-6.05
}

\begin{abstract}
The purpose of the paper is to expose the influence that Latin had on the punctuation system used by Antonio de Nebrija, both in his works written in Latin and those in Spanish. First, an outline is drawn of the evolution of punctuation from Antiquity to the Middle Ages; then, the punctuation used in the three editions of Nebrija's paragraph $D e$ punctis clausularum is compared. Finally, the main conclusions are summarized, including the need to increase and modify the binary system considered by Nebrija as a role model.
\end{abstract}

Keywords: diachronic punctuation, Nebrija's binary system, De punctis clausularum.

Palabras clave: la puntuación diacrónica, el sistema binario de Nebrija, De punctis clausularum.

\section{1.}

\section{Introducción: desde la Antigüedad hasta la Edad Media}

Los signos de puntuación, en la escritura más antigua, no existían. Ahora su cantidad ha aumentado notablemente. Su función puramente prosódico-retórica, con el tiempo, cedió paso a la función

1 Universidad Nicolás Copérnico de Toruń, Facultad de Humanidades, c./ Bojarskiego 1, 87-100 Toruń, Polonia, anetapawlak@umk.pl. 
prevalecientemente sintáctico-semántica, por lo que hoy en día se usan de un modo muy distinto a como se usaban en un principio. Además, los comienzos de los signos de puntuación no parecían idóneos, puesto que, como resalta Delgado García (2017: nota 8), en la Antigüedad clásica, las funciones de los signos estaban poco definidas y su uso carecía de sistematicidad. Por lo tanto, el esbozo de la evolución histórica de la puntuación podría resumirse con las siguientes palabras de Sebastián Mediavilla (2000: 12):

De los griegos tomaron los latinos los principales valores por los que se regiría la puntuación; pero, a lo largo de la Edad Antigua, la Edad Media, e incluso durante el Siglo de Oro, los mismos términos estaban cargados de polisemia y las funciones que se les adjudicaban eran también cambiantes. Además, una era la teoría, y otra era la práctica que seguían, incluso los propios redactores de las normas de puntuación.

No obstante, la necesidad de usar los signos de puntuación, el interés por describirlos y la preocupación por enseñarlos han acompañado a los gramáticos desde los primeros tratados ortográficos. Así pues, en la época clásica grecolatina, la finalidad de los signos de puntuación era la modulación que, como aclara Serna Serna (2011: 239), consistía en "proporcionar unas pausas rítmicas que facilitasen la oratoria y la retórica". En consecuencia, la puntuación en la lengua escrita era lo mismo que las pausas en la lengua hablada, dando así la posibiblidad de recuperar el aliento a quien leía y de proseguir con fluidez la lectura, pero al mismo tiempo procurando que el oyente comprendiera el sentido de lo que se leía.

Por consiguiente, el texto, según las prescripciones de la retórica, se dividía en así llamados en latín periodi, o sea, períodos, que se caracterizaban por ser "de estructura correctamente articulada, con una gran cohesión y un sentido completo" (Serna Serna, 2011: 239). Los períodos, en cambio, podían dividirse en secuencias menores que permitían al orador hacer una pausa breve para respirar sin perder el ritmo ni la fluidez durante la lectura del texto. Esas secuencias, a su vez, se dividían en dos tipos de párrafos: 
los primeros, más largos, se denominaban cola; y los otros, más cortos, se llamaban commata. Así concebían el texto Quintiliano y otros gramáticos del Imperio, por ejemplo, Donato, Prisciano o Diomedes, y así lo transmitían y promulgaban, puesto que, aunque la doctrina de la puntuación era grecolatina en su origen, en su tradición era enteramente latina y en latín (Serna Serna, 2011: 239-240, nota 6).

Por lo demás, cabe señalar que, como hacía falta marcar gráficamente las pausas y las inflexiones de la voz, "los gramáticos relacionaron aquello que originariamente pertenecía a la retórica con un sistema de distinctiones triádico - basado en el periodus, la cola y la commata - y las señales gráficas que las representaban, denominadas positurae" (Serna Serna, 2011: 240). Así pues, el sistema de puntuación clásico, como el nombre de sistema de distinctiones triádico indica, comprendía solamente tres señales, que se colocaban a continuación de la palabra, indicando así la necesidad de hacer cierto tipo de pausa o entonación. Siguiendo a González Pascual (2009: 59), Serna Serna (2011: 245) y Marcos García (2017: 11), esas señales eran:

- un punto en la parte superior [-], denominado distinctio, significaba fin de un período y equivale a nuestro punto y aparte contemporáneo;

- un punto en medio [-], llamado media distinctio, indicaba intervalo medio, lo que hoy en día indica el punto y coma;

- un punto en la parte inferior [.], conocido como subdistinctio, marcaba intervalo breve, por lo que corresponde a la coma actual.

Ahora bien, aunque ese sistema triádico fue propuesto por Dionisio de Tracia, posteriormente fue transmitidito por los gramáticos latinos Diomedes y Donato (González Pascual, 2009: 59). Por otra parte, cabe también tener en mente que, como apunta Serna Serna (2011:240-241), las tres señales de puntuación:

[...] se comenzaron a utilizar en época romana tardía, a partir del siglo II, y no siempre tuvieron una aplicación rigurosa, pues parece que ésta quedaba reservada obligatoriamente para los manuscritos más solemnes. Con el tránsito de la Antigüedad 
a la Edad Media, el sistema de puntuación romano se fue devaluando y se produjo una anarquía en la utilización de espacios en blanco, en el empleo de puntos y se establecieron signos nuevos, como la vírgula. Un hecho constatado es que, ya en la Alta Edad Media, se habían olvidado las técnicas de la retórica, el origen del propio sistema de puntuación [...] y las normas de los gramáticos. No obstante, a pesar de ese abandono, motivado a raíz de los cambios gráficos, lingüísticos y culturales del momento, los gramáticos medievales intentaron conservar, actualizar y poner en práctica esas normas de la oratoria que tan lejos quedaban en el nuevo ambiente del medievo. Así, por ejemplo, autores como Casiodoro, San Jerónimo o San Isidoro recogieron el sistema clásico de puntuación de las tres positurae vel distinctiones obligatorias, logrando mantener viva la tradición latina de la retórica en los primigenios scriptoria monacales. A través de sus Artes dictandi y punctandi medievales se consumó la identificación de los cola y commata con unos determinados signos -entre los que desempeñaban un papel fundamental las vírgulas-, aunque con diferencias, según los autores. De ahí que no sea siempre fácil de interpretar con seguridad el significado de unas mismas denominaciones: división de la frase, pausa, entonación o mero signo gráfico.

Además, como advierte Serna Serna (2011: 241), “aunque las obras sobre la puntuación medieval arrancan de los siglos IV-VI, no será hasta la Baja Edad Media y principios de la Edad Moderna cuando se conceda una mayor importancia a la puntuación". No obstante, los textos medievales dotados de la puntuación presentan dos características que dificultan su análisis contemporáneo. Según Serna Serna (2011: 243), la primera dificultad es una gran variedad de signos de puntuación; y la otra, la falta de una norma predeterminada de su empleo, puesto que su utilización resulta "un tanto aleatoria y arbitraria, en función del tipo de texto, usos escriturarios, particularismos gráficos y geográficos, etc". 


\section{2.}

\section{La puntuación en las obras de Nebrija}

Lo primero que cabe destacar es que Antonio de Nebrija no describió las normas de puntuación ni en su Gramática de la lengua castellana del año 1492 ni en las Reglas de Orthographía del año 1517 , aunque ambas obras fueron escritas en castellano con el fin de presentar las características y las reglas de uso de dicha lengua romance. Por tanto, puede extrañar la ausencia de la mención alguna de la puntuación. No obstante, este hecho tiene una buena explicación que aludimos tras Santiago (1996: 273):

[...] la puntuación castellana, como la puntuación romance en general, y también la usada en otras lenguas no era, en último término, sino una adaptación de la usada en latín, cosa que, ciertamente, no dejaron de reconocer también varios autores, tanto en el siglo XVI como en el XVII. Cabe deducir que solamente por este motivo redactó Nebrija su doctrina sobre la puntuación precisamente en latín y no consideró necesario hacer una nueva versión en castellano para incluirla, por ejemplo, en las Reglas, impresas evidentemente tiempos después.

Así pues, como vemos, Antonio de Nebrija, fiel a la tradición latina, unos años antes que las obras arriba mencionadas, en 1481, publicó en latín y sobre el latín el tratado titulado Introductiones latinae ${ }^{2}$. Como apunta Fernandes (2006: 254), este tratado es también el primer incunable conocido por nosotros con el que se introdujo la imprenta en España. Fue impreso en Salamanca con mil ejemplares que se agotaron inmediatamente. La primera versión de las Introductiones latinae tuvo tres ediciones que

2 Como aporta Fernandes (2006: 257-258), los gramáticos que más influyeron en esta obra de Nebrija fueron Donato (siglo IV d. C.) y su discípulo, Prisciano (siglos V y VI d. C). Por tanto, Nebrija se basa en el latín literario clásico y consituye una gramática normativa del latín, puesto que "la norma emana del uso que de la lengua han hecho los autores clásicos" (Codoñer, 1981: 105, citado por Fernandes, 2006: 258). 
datan de los años 1481, 1482 y 1483 . No obstante, como recoge Santiago (1996: 274), el párrafo dedicado a la puntuación se introduce no antes que en las siguienes publicaciones, que datan de los años "1502 (Sevilla, impresa por Joan Pegnitzer y Magnus Herbst), 1503, 1508 y 1513 (Logroño, por Arnao Guillén de Brocar, el impresor de las Reglas en Alcalá de Henares)". Estas cuatro ediciones se publicaron en vida del gramático, quien muere en 1522. No obstante, como avisan Santiago (1996:274) y Fernandes (2006: 256), antes de su muerte, Nebrija prepara la siguiente edición de las Introductiones latinae, que se publica póstumamente en los años 1523, 1525, 1528, 1530 y 1533 en Alcalá de Henares y, posteriormente, en 1540, 1552 y 1558, en Granada, en la imprenta de los hijos de Nebrija.

Ahora bien, volviendo al párrafo dedicado a la puntuación, este aparece a partir de la edición del año 1502 y se titula De punctis clausularum. A continuación reproducimos su facsímil del año 1533 (Nebrija, 1533: 238):

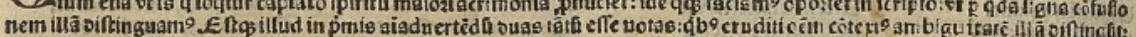

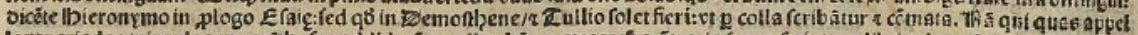



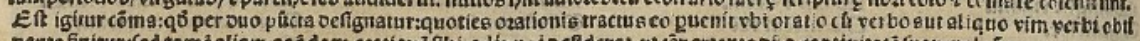

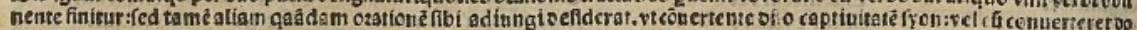

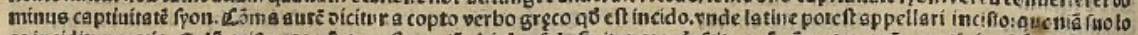

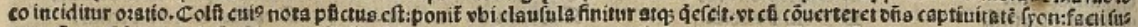



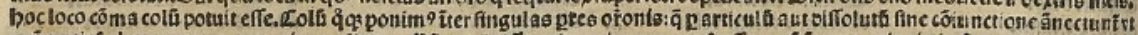

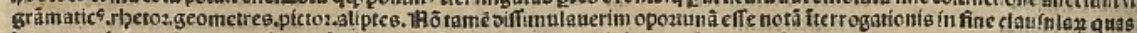
interrogatiuc sut interrogatione admitatiue pferim?, vt bic pietatio bousos:ficnos in fcsptro reporis? $p$ ectermittere vero nul

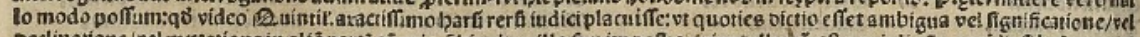



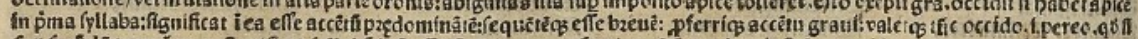



Como podemos percibir, la extensión del párrafo no es grande. Al contrario, es brevísima. No obstante, resulta curioso observar que en un texto tan corto la puntuación empleada fuera cambiando de edición en edición, alejándose sucesivamente de la puntuación original aplicada por Nebrija en la edición de $1502^{3}$.

3 De ello era también plenamente consciente el mismo Nebrija. Como advierte Santiago (1996: 274), en el colofón de las ediciones de Logroño de 1508 y 1513 "se dice que corrigió el texto el propio Nebrija, aunque también se deja constancia allí de que podrían habérsele pasado por alto (a él o a quienes en el curso de la impresión 
Para ilustrarlo, cotejamos a continuación tres ediciones diferentes de las tres primeras oraciones del párrafo De punctis clausularum provenientes respectivamente de los años 1502, 1533 y 1558.

Así pues, en la edición del año 1502, 14 veces aparecen los signos de puntuación, que son dos, y que marcamos en negrita en la siguiente transcripción ${ }^{4}$ :

Quemadmodum in sermonis ductu necesse est fieri quasdam silentii distinctiones: tum ut auditor intelligat clausularum diversitatem: tum etiam ut is que loquitur: captato spiritu maiori acrimonia pronunciet: idem quoque faciamus oportet in scripto: ut per quaedam signa confusionem distinguamus. Estque illud in primis animadvertendum duas tantum esse notas: quibus eruditi omnem contextus ambiguitatem illam distinguunt: dicente Hieronymo in prologo Esaiae sed quod in Demosthene et Tullio solet fieri: ut per cola scribantur et commata. Nam qui quas appellant periodos. virgulas et parentheses addiderunt: nullos habent auctores: cum e contrario sacrae scripturae libri colo et commate contenti sint.

hicieron sus veces) «algunas letras o algunos puntos»: «ab eodem autore [sic] ad ammussim [sic] castigata nisi quatenus aliquae litterae aut puncta, oculos illius vel eorum qui cum imprimerentur suas gesserunt vices lineas percurrentium, potuerunt effugere»".

4 Tanto la transcripción del año 1502 como las de los años 1533 y 1558, que analizamos en el presente trabajo, vienen del artículo de Santiago (1996: 282-283), quien presenta la siguiente traducción libre del latín al español de dicho fragmento: "En sustancia, pues, la puntuación es a la lengua escrita lo que las pausas a la hablada, en la que, además de facilitar al oyente la comprensión de lo que se dice, dan la posibilidad al que habla de recuperar el aliento y proseguir con nueva energía. Los que saben de esto, los «enseñados», sólo emplean dos signos de puntuación, según advierte San Jerónimo, en el prólogo de Isaías, respecto de los textos de Demóstenes y Cicerón y también los de las Sagradas Escrituras, que se ordenan únicamente según colos y commas. En cambio quienes a este sistema añaden períodos, vírgulas y paréntesis carecen de autoridades en que apoyarse" (Santiago, 1996: 276). 
Como vemos, los signos de puntuación que aparecen aquí son el colum, representado por el punto (.) y empleado cuatro veces, y la comma, indicada por dos puntos (:) y usada diez veces. Eso es así, porque el sistema de puntuación empleado por Nebrija era precisamente binario, o sea, compuesto por tan solo dos signos: el colum (.), que señalaba que la oración ya había concluido, pero también se ponía para separar palabras enumeradas sin conjunción copulativa; y la comma (:), que se introducía al final de una oración con verbo si se deseaba añadir otra oración a continuación para separar las partes constituyentes de una oración compuesta. No obstante, el mismo Nebrija admitió que a veces podían darse contextos en los que no se sabía si poner la comma o el colum, por lo que el sistema binario empezó a ser deficiente. No es extraño, por tanto, que ya en la edición de 1533, aparecida después de la muerte del filólogo, que tuvo lugar en 1522, se utilizara un sistema de puntuación ampliado. Fijémonos ahora en la siguiente transcripción de las tres primeras oraciones del párrafo De punctis clausularum, en la que al lado de la puntuación de la edición del año 1502 ponemos también en negrita, pero entre paréntesis los signos de puntuación de la edición del año 1533:

Quemadmodum in sermonis ductu necesse est fieri quasdam silentii distinctiones:(:) tum ut auditor intelligat clausularum diversitatem:(:) tum etiam ut is que loquitur:( $)^{5}$ captato spiritu maiori acrimonia pronunciet:(:) idem quoque faciamus oportet in scripto:() ut per quaedam signa confusionem distinguamus.(.) Estque illud in primis animadvertendum duas tantum esse notas:(:) quibus eruditi omnem contextus ambiguitatem illam distinguunt:(:) dicente Hieronymo in prologo Esaiae(:) sed quod in Demosthene(/) et Tullio solet fieri:(:) ut per cola scribantur et commata.(.) Nam qui quas appellant periodos.(/) virgulas(/) et parentheses addiderunt:(:) nullos habent auctores:(:) cum e contrario Sacrae Scripturae libri colo et commate contenti sint.(.)

5 Los paréntesis vacíos designan la falta del signo de puntuación. 
Al comparar cuantitativamente las dos versiones, podemos notar que en la edición del año 1533 aumenta la aparición de los signos de puntuación hasta 15 veces, pero también aumentan sus representantes gráficos hasta tres. Estos tres signos son: el colum (.), usado tres veces, la comma (:), empleada nueve veces, y la vírgula (/), en forma de una línea oblicua, hoy conocida como la barra. La vírgula, como novedad, aparece tres veces, de las cuales una vez como sustituto del colum en su función de separar enumeraciones y dos veces delante de la conjunción et, también con la función de señalar enumeraciones.

No obstante, muy pronto la vírgula queda sustituida en grafía por la forma que hoy en día conocemos como coma, lo que ya queda ilustrado en la última edición de las Introductiones del año 1558, impresa en Granada por los hijos de Nebrija. A continuación, reproducimos las tres primeras oraciones del párrafo De punctis clausularum en el que 19 veces aparecen tres signos de puntuación que ponemos en negrita, entre corches, al lado de la puntuación del año 1502 y del año 1533:

Quemadmodum in sermonis ductu necesse est fieri quasdam silentii distinctiones:(:)[,] tum ut auditor intelligat clausularum diversitatem:(:)[,] tum etiam ut is[,] que loquitur:()[,] captato spiritu maiori acrimonia pronunciet:(:)[:] idem quoque faciamus oportet in scripto:()[,] ut per quaedam signa confusionem distinguamus.(.)[.] Estque illud in primis animadvertendum duas tantum esse notas:(:)[,] quibus eruditi omnem contextus ambiguitatem illam distinguunt:(:)[,] dicente Hieronymo in prologo Esaiae(:)[.] sed quod in Demosthene(/)[,] et Tullio solet fieri:(:)[,] ut per cola scribantur et commata.(.)[.] Nam qui[,] quas appellant periodos.(/)[,] virgulas(/)[,] et parentheses addiderunt:(:)[,] nullos habent auctores:(:)[,] cum e contrario Sacrae Scripturae libri colo et commate contenti sint.(.)[.]

Los tres signos de puntuación empleados aquí son, pues, la vírgula (,) que ya tiene la misma grafía que la coma actual y que aparece hasta trece veces, la comma (:), que en esta ocasión aparece solamente una vez, relegada por la abrumadora presencia de 
la vírgula, y el colum (.), usado cuatro veces, por lo que su empleo parece más estable que el de las demás grafías presentes en las tres ediciones analizadas, que, recordemos, desde 1502 hasta 1558 abarcan el período de cincuenta y seis años.

\section{3.}

\section{A modo de conclusión}

Tomando en consideración todo lo presentado, nos proponemos extraer las siguientes conclusiones.

En primer lugar y volviendo a la Antigüedad, cabe subrayar, siguiendo a Santiago (1996: 278), que, "la tríada cola-commata-periodus, presente ya al menos desde Probo y Quintiliano, es mucho más frecuente en los autores antiguos (latinos) y medievales que la díada cola-commata, que tiene como autoridad más relevante entre los latinos a Prisciano". Por tanto, parece evidente que la ausencia del periodus, pero también de las vírgulas y los paréntesis en el sistema de puntuación de Nebrija testimonia su gran inclinación hacia las enseñanzas de Prisciano.

En segundo lugar, hay que recalcar que la escasa presencia de los signos de puntuación en la gramática latina nebrisense no significa que no hubiera más signos de puntuación. Por ejemplo, Nebrija acepta el signo de interrogación, que gráficamente aparece solo en forma de cierre al final del enunciado, pero no lo incluye al lado de la comma y el colum. En cuanto a los dos signos rechazados por Nebrija, vírgulas y paréntesis, estos se usan comúnmente en la imprenta. Recordemos que, en el párrafo De punctis clausularum, en las ediciones póstumas de los años 1533 y 1558, se percibe la presencia de las vírgulas, pero en las ediciones vigiladas por Nebrija no fueron admitidas.

En tercer lugar, queda incuestionable que Nebrija aplica el sistema binario de puntuación también a la lengua castellana, para la cual ya no elabora unas normas de puntuación distintas a las latinas. Esto no quiere decir que pusiera menos atención a la lengua castellana. En absoluto. A sabiendas de que el castellano no era, en su origen, sino una de las variantes dialectales del latín, 
fue precisamente Antonio de Nebrija quien notablemente contribuyó a la conversión del castellano en la lengua oficial de todo el Estado, con la publicación en 1492 de su Gramática de la lengua castellana ${ }^{6}$.

En cuarto lugar y fijándonos de nuevo en las Introductiones latinae de Nebrija, es necesario señalar que al comparar la puntuación empleada en el párrafo De punctis clausularum procedente de las ediciones de los años 1502, 1533 y 1558, nos encontramos con una tendencia al crecimiento de la frecuencia de aparición de los signos de puntuación, puesto que partiendo de las 14 veces que se emplean en 1502, pasando por los 15 en 1533, llegamos hasta la edición de 1558, donde se emplean los signos de puntuación 19 veces. Además, su cantidad y representantes gráficos también

6 Merece la pena citar dos fragmentos del prólogo a la Gramática de la lengua castellana dedicado a la reina Isabel la Católica, que ilustran el empeño de Nebrija en divulgar el castellano mediante su obra, pero también ejemplifican la presencia del colum y de la comma como únicos signos de puntuación aplicados en su redacción: "I será necessaria una de dos cosas: o que la memoria de vuestras hazañas perezca con la lengua: o que ande peregrinando por las naciones estranjeras: pues que no tiene propria casa en que pueda morar. En la çanja de la cual io quise echar la primera piedra. y hazer en nuestra lengua lo que Zenódoto en la griega y Crates en la latina. Los cuales aunque fueron vencidos de los que después de ellos escriuieron: a lo menos fue aquella su gloria y será nuestra: que fuemos los primeros inuentores de obra tan necessaria. lo cual hezimos en el tiempo más oportuno que nunca fue hasta aquí.

[...] Que después que vuestra Alteza metiesse debaxo de su iugo muchos pueblos bárbaros y naciones de peregrinas lenguas: y con el vencimiento aquellos ternían necessidad de recebir las leies: quel vencedor pone al vencido y con ellas nuestra lengua: entonces por esta mi Arte podrían venir en el conocimiento della como agora nos otros deprendemos el Arte de la Gramática latina para deprender el latín. y cierto assí es que no sola mente los enemigos de nuestra fe que tienen ia necessidad de saber el lenguaje castellano: mas los vizcaínos. navarros. franceses. italianos. y todos los otros que tienen algún trato y conversación en España y necessidad de nuestra lengua: si no vienen desde niños a la deprender por uso: podrán la más aína saber por esta mi obra”. 
tienden a cambiar, por ejemplo, en 1502 estamos ante dos signos, que son el colum (.) y la comma (:), pero en 1533 estamos ya ante tres signos, que son el colum (.), la comma (:) y la vírgula (/). En cambio, en 1558, estos tres signos conservan su cantidad, pero la vírgula (,) reviste nueva grafía, dando comienzo a la coma actual.

En quinto lugar, podemos observar que el empleo de los signos de puntuación todavía carece de estabilidad normativa, por lo que prácticamente se basa en las costumbres escriturarias latinas del mismo Nebrija, pero también de los impresores que editan posteriormente sus obras. Recordemos, por tanto, que el lugar de la imprenta de las Introductiones latinae es diferente para las tres ediciones, a saber, Sevilla en 1502, Alcalá de Henares en 1533 y Granada en 1558.

En último lugar, cabe destacar que la tradición latina de puntuación de Nebrija no tuvo mucho éxito, ya que casi diez años después de su muerte, en 1531, se publicó en castellano el Tractado de orthographia de Alejo Venegas. Como hace notar Delgado García (2017: 7), este autor, básandose también en el legado latino, a la comma (:) y al colum (.) añade cuatro señales de puntuación más, que son el articulus (:), los paréntesis [()], la vírgula (/) y el interrogante (?), por lo que la necesidad de la modificación y del desarrollo del sistema binario nebrisense resultó inevitable, pero no solamente en cuanto a sus representantes gráficos, sino también en cuanto a su empleo y su función. Pero este tema ya es harina de otro costal.

\section{Bibliografía}

Delgado García, M. (2017): "La puntuación en documentos novohispanos. Una mirada descriptiva”. Anuario de Letras. Lingüistica y Filología, Vol. V, sem. 1, año 5-36.

Fernandes, G. (2006): “As Introductiones latinae (1481) de Élio António de Nebrija”. Humanitas 58: 253-271.

González Pascual, B. (2009): Edición y estudio de la 'Orthographía española' de la Real Academia Española (1741). Antecedentes y consecuentes. Tesis doctoral dirigida por Manuel Peñalver Castillo. Universidad de Almería: [en línea] <https://books.google. $\mathrm{pl} /$ books?id=KTtBAQAAQBAJ $>$ [20.05.2019]. 
Marcos García, J.-J. (2017): Fuentes para Paleografía. Manual de usuario. $5^{\mathrm{a}}$ edición 6-Enero-2017. Plasencia (Cáceres): [en línea] $<$ http://guindo.pntic.mec.es/ jmag0042/manual_paleograf. pdf $>$ [23.06.2019].

Nebrija, A. de (1492): Gramática de la lengua castellana, [en línea] $<$ http://www.antoniodenebrija.org/prolog o.html > [28.06.2019].

Nebrija, A. de (1533): Introductiones latinae (Commentaria introductionum in grammaticam Latinam). Alcalá de Henares: Miguel de Erguía, 30 septiembre 1533 [en línea] <http://corpusnebrissense.com/caracola/introductiones/intr1533.html $>$ [23.09.2019].

Santiago, R. (1996): "La puntuación según Nebrija". DICENDA. Cuadernos de Filología Hispánica, n. 14: 273-284.

Sebastián Mediavilla, F. (2000): La puntuación en el Siglo de Oro: teoría y práctica. Tesis doctoral dirigida por Francisco Rico Manrique. Universidad Autónoma de Barcelona: [en línea] $<$ http://hdl.handle.net/10803/4855> [13.06.2019].

Serna Serna, S. (2011): "El Becerro Gótico de Cardeña: signos de puntuación”, en: E.E.

Rodríguez Díaz y García Martínez, A.C. (eds.), La escritura de la memoria: los cartularios, VII Jornadas de la Sociedad Española de Ciencias y Técnicas Historiográficas, Universidad de Huelva: 237-254. 